Classification

Physics Abstracts

$73.40-73.25-73.30-72.20 \mathrm{~F}$

\title{
Geometrical factor in multi-interface homostructures
}

\author{
Z. T. Kuznicki ( $\left.{ }^{1}\right)$, A. Martinez $\left({ }^{2}\right)$, J.-C. Muller $\left({ }^{1}\right)$ and P. Siffert $\left({ }^{3}\right)$ \\ (1) Centre de Recherches Nucléaires (IN2P3), Laboratoıre PHASE (UPR du CNRS $\mathrm{N}^{\circ}$ 292), \\ 67037 Strasbourg Cedex 2. France and \\ Université Louis Pasteur, Département de Physique, 3 rue de l'Université, 67000 Strasbourg, \\ France \\ (2) Institut National des Sciences Appliquées de Toulouse, av. de Rangueil, 31077 Toulouse \\ Cedex. France and \\ Laboratoire d'Automatique et d'Analyse des Systèmes du CNRS, 7 av. du Colonel Roche, 31077 \\ Toulouse Cedex, France \\ ( $\left.{ }^{3}\right)$ Centre de Recherches Nucléaires (IN2P3), Laboratoire PHASE (UPR du CNRS N²92), \\ 67037 Strasbourg Cedex 2, France
}

(Received 2 October 1992, revised 26 March 1993 and 13 January 1994, accepted 24 February 1994)

\begin{abstract}
In an experimental investigation carried out on multilayer silicon homostructures (of the $n^{+}-n-n^{+}$type) an electric interaction between the two homointerfaces through majority carriers as a function of the geometrical factor has been shown. The two model homointerfaces were formed in the same monocrystal (CVD epitaxy) by only an abrupt change in the impurity doping level. Samples differing in the spacing of their parallel interfaces were characterized in the stationary and steady states (static and quasi-static regimes) to reveal modifications in their macroscopic transport. It has been demonstrated that the thermionic emission current, normally taken into account in the barrier transport (with large potential barriers) dominates in only two of five characteristic bias intervals. The two other phenomena, also related to the geometrical factor, i.e. the diffusion-drift and tunneling currents, dominate the conduction over practically the whole dc-bias range. These results allow the study of short and long range electrical interactions as well as the free carrier micromovement of simple and complex semiconductor interfaces of multilayer devices.
\end{abstract}

\section{Introduction.}

Semiconductor interface regions have diverse effects on the operation of semiconductor devices. For example, an abrupt change in doping level alone causes local changes in the carrier concentration in its neighborhood with a width of one extrinsic Debye length [1, 2] which is sufficient for modification of carrier scattering mechanisms, recombination velocity 
or excess carrier lifetime [3,4]. The problem of two low-high (L-H) type homointerfaces relatively near each other was recently studied in [5-8]. This analytical approach allows a physical interpretation of the barrier limited conduction (thermionic current) which is wellknown from self-consistent numerical simulations. The steady state current-voltage characteristics of majority carriers through two identical interfaces can be dominated by any of several phenomena : diffusion-drift, thermionic emission, tunneling or ballistic transport [8]. A more subtle analysis of the static characteristics has to take into account, in certain cases, not only the height of the potential barrier next to the interface but also the barrier modifications caused by an external electric disturbance $[9,10,6,11]$.

Two near interfaces can create interactions : i) of short-range of the unipolar type (majority carriers) in symmetrical and asymmetrical barriers $[12,5]$ or ii) of long-range of the bipolar type (minority carriers) occurring in diffusion length and carrier lifetime [13]. All of our investigations correspond to the theoretical model of the abrupt L-H homointerface built in a multilayer structure $[5,12]$. In the steady-state all samples were therefore studied over a wide range of biases (lying between a hundredth and hundreds of volts [8]).

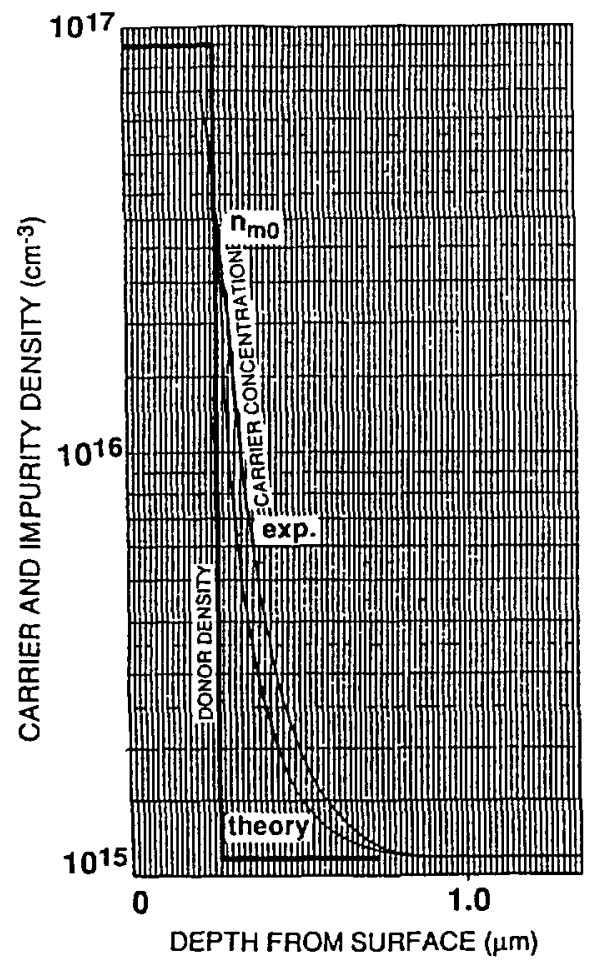

a)

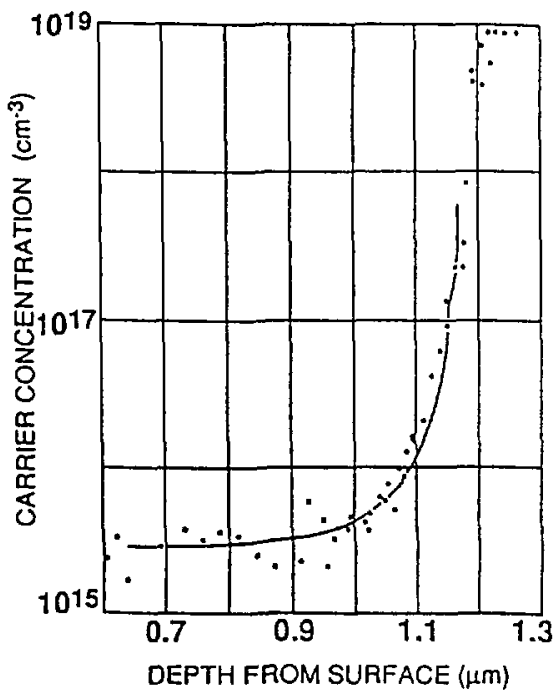

b)

Fig. 1. - Majority carrier profiles at simple abrupt L-H homointerfaces : a) comparıson of experimental (thick line) [44] and theoretical (thin line) $[25,14]$ distributions. The two curves were normalized at the interface carrier concentration $n_{\mathrm{m} 0}[20]$ (doping ratio $N=N_{\mathrm{DH}} / N_{\mathrm{DL}}=81.82$ ); b) comparison of C-V (contınuous line) and spreading resistance (dots) results obtained with correction (doping ratio $N=3.103 \times 10^{3}, N_{\mathrm{DL}}=2.9 \times 10^{15} \mathrm{~cm}^{-3}$ ) where the upper layer is the lightly doped one [44]. This example demonstrates the penetration limitation of the non-destructive $\mathrm{C}-\mathrm{V}$ method in a highly doped region. 


\section{Electric interaction at the homointerface.}

In a one-dimensional semiconductor structure, the geometrical factor ( $\left.{ }^{1}\right)$ depends principally on two kinds of parameters, namely the doping levels and the thickness of the superimposed layers and especially the thickness of the inserted lightly doped layer(s). Firstly it concerns the simple interface transition zone. The actual distributions of free-carriers, electric field or electrostatic potential are directly affected by the extension of this zone $[14,15]$. Secondly the geometrical factor involves the electric interactions between interfaces of a multilayer structure. In figures 2 and 3 there are many barrier shapes differing from each other only by the two L-H homointerface spacings [5]. The geometrical factor effect can be analyzed from many points of view : i) in the stationary-state with its potential (quantum) well aspects and in the steady-state with potential barrier modification. The homo- and heterointerfaces created inside the semiconductor volume act with or without direct relation to the intrinsic properties of the material composing the structure

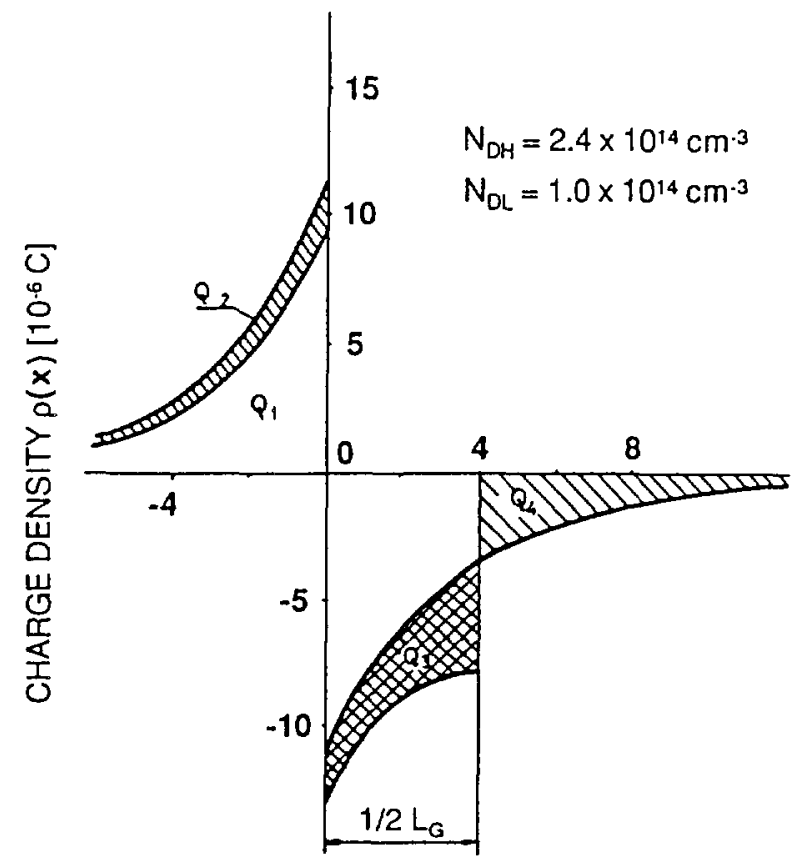

\section{DISTANCE FROM THE INTERFACE $\times\left[10^{-5} \mathrm{~cm}\right]$}

Fig. 2. - Space charge evolution in the case of a small interface spacing: $Q_{1}$ is the actual total charge in the high region, $Q_{2}$ is the total charge blocked inside the high region by the action of a second interface, $Q_{3}$ is the supplementary total charge inside the low region and $Q_{4}$ is the total charge not distributed far inside the low region [5].

(1) The geometrical factor represents the influence on the electrical properties of only the geometrical dimensions (the thickness for example) of a given semiconductor region. It is important for relatively small dimensions comparable to or smaller than the extrinsic Debye length. 


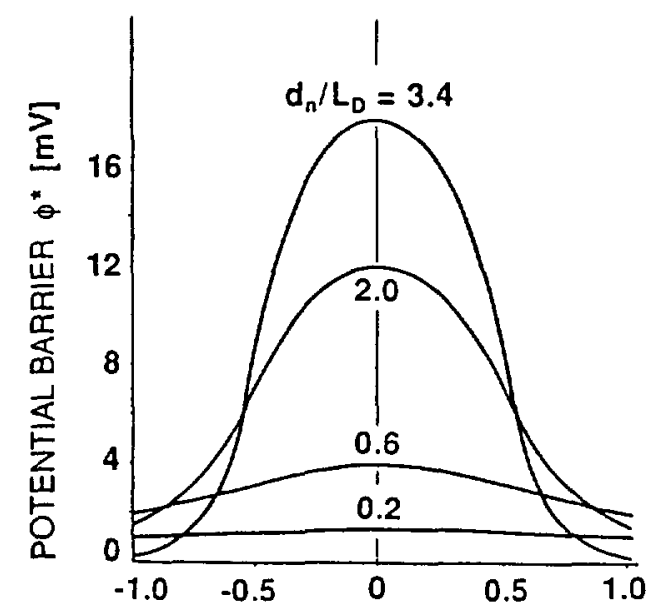

a)

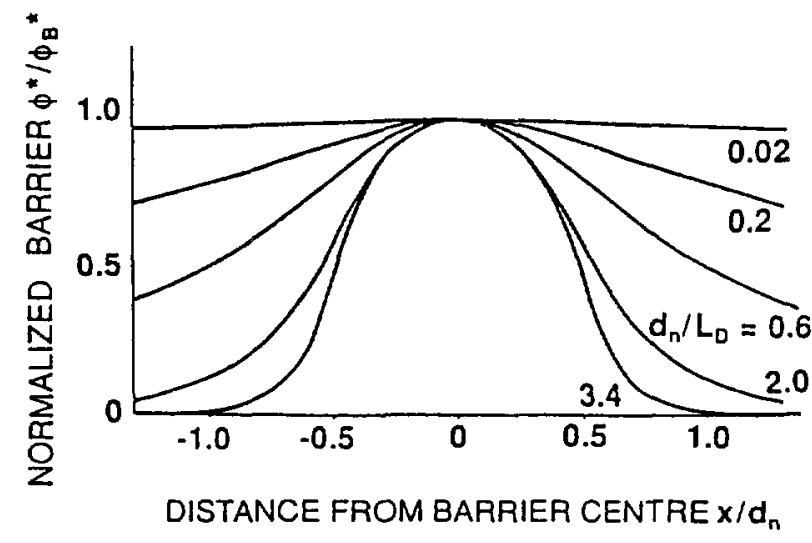

b)

Fig. 3. - Relative changes in the shape of the two-Interface potential barrier as a function of the interface spacing $d_{\mathrm{n}}$; potential without (a) and with (b) normalization. The ratio of interface spacing to the extrunsic Debye length $d_{\mathrm{n}} / L_{\mathrm{D}}$ is taken as parameter [5].

One of the basic features of steady-state transport through multilayer structures is its dependence on a geometrical factor. The steady-state current-voltage characteristic of a simple $\mathrm{L}-\mathrm{H}$ homojunction is mainly nonlinear [16-18, 8]. This macroscopic behavior is not still sufficiently explained, especially in a regime of intermediate and high injection levels or in the case of space limited structures [19]. Boundary conditions of the junction interface are determined by the electrostatic law $[15,20]$, and entire distributions in any structure can be found numerically [21-23]. For a semi-infinite or space limited structure, these distributions can also be found either through the recent analytical [24-26, 14] or numerical [6, 7] solutions of Poisson's equation. The corresponding experimental profiles of majority carriers and doping impurities are obtained from $\mathrm{C}-\mathrm{V}$ or spreading resistance measurements.

2.1 SHORT-RANGE INTERACTIONS. - In the usually assumed multilayer geometry, the conduction carriers traverse a series of at least two interfaces perpendicularly, which in addition are 
close to each other. Closeness enhances the nonlinearity that cannot be accounted for by considerations published so far. The bipolar transport (forward current-voltage characteristics) through a planar abrupt structure with two homojunctions $\left(\mathrm{p}^{+}-\mathrm{n}-\mathrm{n}^{+}\right.$structure) has been analyzed in [10]. The curves, derived from the basic current and continuity equations, extend the analysis from the low injection range continuously to the high injection region. The minority carrier distributions as well as the related field and current distributions have been shown.

The corresponding unipolar transport of two similar homojunctions $\left(n^{+}-n-n^{+}\right.$structure) has been analyzed in [6-8] within the macroscopic properties of non-ballistic transport. Particular aspects of the ballistic transport has been published in [27-29]. Except for a few papers (e.g. of Kim and Yang [6] and van der Ziel's group [7]) any treatment of the injection levels of the collision transport $[30,12,8]$ is absent from the literature. According to the thermionic emission model (presented in its numerical aspect in [7] and in its analytical aspect in our earlier paper [8]), it is possible to separate macroscopic components of the conduction current in the steady-state in the case of a thin structure with the spillover effect.

2.2 LONG-RANGE INTERACTIONS. - It is an interesting feature of a thick structure without spillover effect that the actual interface interaction is much more extended than the junction thickness [11] (or the extrinsic Debye length [1]). The experimental and the numerical analyses $[3,4,31]$ show that the $\mathrm{L}-\mathrm{H}$ interface $\left(\mathbf{n}-\mathbf{n}^{+}\right.$or $\mathrm{p}-\mathrm{p}^{+}$structure $)$can extend its activity inside the low region well beyond the corresponding space charge penetration. It influences the recombination properties of the minority population over many extrinsic Debye lengths. This problem is studied especially within back surface field (BSF) solar cells and will be analyzed in detail in a subsequent publication [32].

\section{Homointerfaces in the stationary-state - geometrical factors.}

3.1 SAMPLE DESIGN AND PREPARATION. - From a practical point of view, our goal was to realize uniformly doped, nearly abrupt short structures (see Fig. 4). To accomplish this, a detailed study of the technological conditions and measurement methods of non-disturbed simple interfaces was undertaken. The chosen multilayer CVD-epitaxy [33] consists of several uniformly doped layers whose thickness varies between fractions and tens of micrometers [34].

The samples have been fabricated on the same substrate in one four-stage process guaranteeing the uniformity of technological parameters [35, 36]. Simultaneously, to enable one-stage non-destructive characterization, the doping levels must be limited so as to allow the complete profile determination in one continuous measurement as by the $\mathrm{C}-\mathrm{V}$ method.

To limit impurity rediffusion (principally from the substrate towards the epitaxied layer) the CVD growth process must be performed as rapidly and at the lowest temperature as possible (below $700{ }^{\circ} \mathrm{C}$ ). For temperatures below $1400 \mathrm{~K}$, Si epitaxy is controlled by reactions occurring at the growing surface where the activation energy is approximately $30 \mathrm{kcal} / \mathrm{mole}$. The growth rate is therefore strongly temperature dependent. Maintaining this high growth rate requires increasing the concentration of $\mathrm{SiCl}_{4}$ in the $\mathrm{H}_{2}$. However, this method suffers from the occurrence of a number of rapidly developing structural imperfections. A CVD-epitaxy that matches these two requirements of impurity profile abruptness and structural perfection has been developed $[33,35]$. Values have been obtained for two major parameters of the process with a theoretical CVD-epitaxy model [33]. Details on this process are given in [36].

3.2 TRANSITION ZONE CHARACTERIZATION : IMPURITY AND FREE-CARRIER PROFILES. - In a perfect multilayer structure, the transition zones between different layers are extremely narrow, i.e. the doping concentration varies abruptly at the interface, $\operatorname{grad} N_{\mathrm{D}}(x) \rightarrow \infty$. Real 


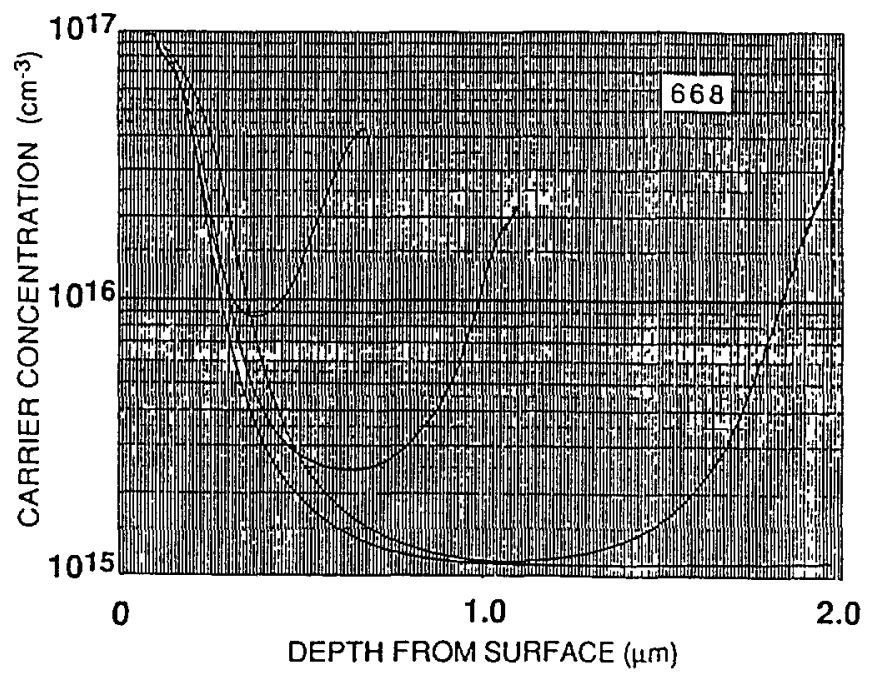

a)

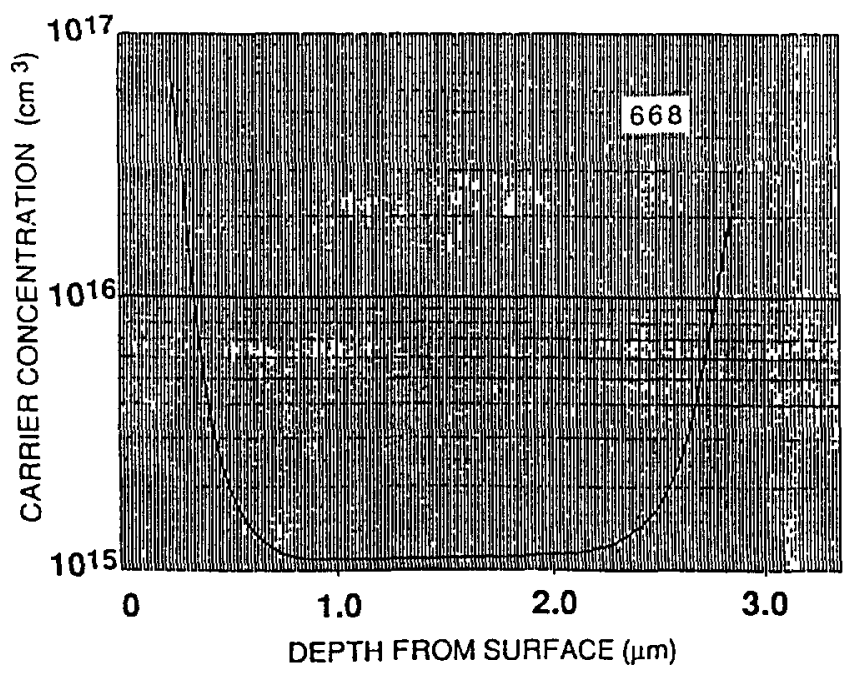

b)

Fig. 4. - Experimental C-V profiles of majority carrier concentrations : a) in three samples whose interface spacings derived from the electrostatic law for space limited structures are : $d_{\mathrm{n}}=0.43 \mu \mathrm{m}$ : $0.86 \mu \mathrm{m} ; 1.70 \mu \mathrm{m}$ [8]. The lower fourth curve that represents a thick sample where $d_{\mathrm{n}}=2.64 \mu \mathrm{m}$ is shown wholly in (b).

transition zones by molecular beam epitaxy (MBE) approach this criterion with one or two atomic layer thicknesses.

Some stationary-state properties can be estimated from the experimental impurity and freecarrier profiles. But in our detailed study the experimental results have to take into account distribution uncertainties. In the vicinity of the L-H interface, the abrupt profile $\left({ }^{2}\right)$, of ionized

(2) In reality graded but with a large impurity gradient. 
impurities $N(x)$ is different from that of the graded majority carrier profile $n(x)$. The relationship between $N(x)$ and $n(x)$ has been developed by Kennedy and O'Brien [21] and substantiated by Nishida [37] with a comment from $\mathrm{O}^{*}$ Brien [38] as

$$
N(x)=n(x)-\left(\frac{k T}{q}\right)\left(\frac{\varepsilon \varepsilon_{0}}{q}\right) \frac{\mathrm{d}}{\mathrm{d} x}\left[\frac{1}{n(x)} \frac{\mathrm{d} n(x)}{\mathrm{d} x}\right]
$$

where $\varepsilon$ and $\varepsilon_{0}$ are the semiconductor dielectric constant and vacuum permittivity respectively and

$$
n(x)=-\frac{C^{3}}{q \varepsilon \varepsilon_{0}}\left(\frac{\mathrm{d} C}{\mathrm{~d} V}\right)^{-1}
$$

This method gives good results in the case of nearly abrupt structures with impurity gradients localized in regions much narrower than the local extrinsic Debye length [39, 40]. But the questions remain what is the significance and accuracy of the electrical profile actually measured. According to Kennedy and co-workers [41, 21] the differential capacitance method developed by them from the conventional Schottky method establishes directly, in the case of really abrupt structures, a majority carrier profile. Their idea is supported by Wilson [40] and partially by Kroemer and Chien [42] who introduced the notion of apparent electron distribution which differs slightly from the actual distribution especially inside the lightly doped region far from the interface. The theoretical resolution is comparable with the extrinsic Debye length of the highly doped region, but not with the one of the lightly doped region. Kennedy's conclusion is contested by Johnson and Panousis [43] who adhere to the opinion that the differential capacitance method at the edge of the depletion region gives neither the doping density nor the free carrier concentration, but an apparent doping concentration slightly smaller than the true impurity density. This contradiction suggests that the subtle electrical composition of the transition zone is not simple and that the resultant $\mathrm{C}-\mathrm{V}$ curve may depend on the actual measuring conditions. Thus our hypothesis of many fraction accumulation [25] may be useful in a resolving of the ambiguity.

In experimental investigations of the space charge profile from the $\mathrm{C}-\mathrm{V}$ distribution we have used the improved method proposed by Kroemer and Chien [42] who suppose that the measured curves represent the resultant profile of the majority carriers and that the impurity profiles can be deduced directly from these curves within Kennedy's method [38]. The detailed analysis of measurements comparing the ideal homointerface distribution [34] confirmed: i) the excellent crystalline and electrical quality of our samples and ii) that the total space charge distribution is a difference of carrier and impurity profiles. The extension of the one transition zone from a highly to a lightly doped region is shown in figure 1 . We selected the electrical interface as reference for both experimental and theoretical carrier profiles using the accurate formula for the interface concentration $n_{\mathrm{m} 0}[20,8]\left(^{3}\right)$. One can estimate that the shift between the experimental and the ideal free carrier profiles is smaller than about $300 \AA$ over two orders of magnitude in doping level ratio. Fortunately, after Shenai and Dutton [26], the real impurity distribution is much more abrupt than can be measured by the traditional $\mathrm{C}-\mathrm{V}$ method. So our single transition zones are abrupt enough to be treated with idealized homojunction theory.

Our three-layer symmetrical structures (both $\mathrm{n}^{+}$-layers were doped identically to $9.0 \times 10^{16} \mathrm{~cm}^{-3}$ whereas every $n$-layer remained at $n=1.1 \times 10^{15} \mathrm{~cm}^{-3}$ ) contain two homointerfaces (see Fig. 4) having different spreading of their structural transition zone : the shallow junction has rather abrupt behavior while the deeper one is more gradual. The

$\left.{ }^{3}{ }^{3}\right)$ The theoretical distributions computed within either Maxwell-Boltzmann or Fermi-Dirac statistics [23] give differences too small to be visible in this figure. 
measuring accuracy for both are different because the signs of the doping gradients. For a decreasing profile, which is easier to determine, precise voltage measurements are required over distances comparable to the local extrinsic Debye length. Large changes in differential capacitance occur as a consequence of the profile shape. The accuracy for measurement of an increasing profile is greater since large voltage excursions are required to deplete the material as doping density increases. Our first junction influences predominantly the steady-state characteristic of a three-layer structure and its structural transition zone has been approximated with a nearly abrupt linear distribution as presented by Wilson [40].

In a numerical model, the shallow junction has a linear transition zone about $200 \AA$ wide whereas the deep junction is more gradual and different for each sample thickness. Corrections for the transition zone thickness of deep junctions whose surplus vary from about $50 \%$ in the case of the thickness n-layer to $10 \%$ for the thinnest $n$-layer (by 20 and $35 \%$ in the two intermediate thickness layers).

Because $\mathrm{C}-\mathrm{V}$ profiling suffers from a relatively small extension into the semiconductor volume, particularly for higher doping levels, these results must be supplemented with those derived from other techniques as the spreading resistance method $[45,46]$ necessarily provided with a correction in the case of submicronic structures [36, 47, 48]. This method, giving the profiles over the whole three-layer structure with its two transition zones, is destructive and must be executed separately on a twin samples [44].

The example of results from both methods for a sample with doping level ratio $N=N_{\mathrm{DH}} / N_{\mathrm{DL}}=3.103 \times 10^{3} \mathrm{~cm}^{-3}$ is shown in figure $1 \mathrm{~b}$. It can be noted that the two methods yield practically the same results inside the lightly doped region, except for the usual scatter of the spreading resistance points which may be smoothed numerically to a useful line for the electric thickness determination.

3.3 SAMPLE THICKNESS-INTERFACE SPACING. - To compute and compare the interface spacing (and as a consequence the corresponding semiconductor volume) from our experimental measurements, it is important to know as precisely as possible the actual electrical interface positions. The thickness of the inserted n-layer especially had to be measured accurately. We have developed a method based on free carrier profiles and electrostatic law conditions assuming that a measurement gives a free carrier profile of the interface $[40,37,41]$; the electric interface of the homojunction is located at the doping interface (where the free carrier concentration equals $n_{\mathrm{m} 0}$ ). It can be determined accurately from the electrostatic law $[15,20]$ as a function of only the doping densities as follows

$$
n_{\mathrm{m} 0} / N_{\mathrm{DH}}=\exp \left[N_{\mathrm{DH}} \ln \left(N_{\mathrm{DH}} / N_{\mathrm{DL}}\right) /\left(N_{\mathrm{DH}}-N_{\mathrm{DL}}\right)+n_{\mathrm{t}}^{2} /\left(N_{\mathrm{DH}} N_{\mathrm{DL}}\right)-1.0\right]
$$

where $N_{\mathrm{DH}}$ and $N_{\mathrm{DL}}$ are the impurity concentrations in the high and low doped regions respectively.

The same accuracy may not be attainable in the determination of whole abrupt multilayer profiles where the conventional $\mathrm{C}-\mathrm{V}$ method yields a distribution with a long tail extending deep into the lightly doped layer as illustrated in figure 1. The assumption is made that an overlap of both impurity and carrier profiles is possible at least in the strict vicinity of the interface. Wilson's [40] detailed study of the apparent free carrier, free carrier and impurity profiles supports this hypothesis.

The interface spacing to be realized has been designed from the macroscopic transport around the spillover phenomenon $[6,7]\left({ }^{4}\right)$, namely from the extrinsic Debye length and the thickness of the free accumulation layer [23] $\left(^{5}\right)$. We chose the following width for the lightly

$\left({ }^{4}\right)$ The spillover phenomenon signifies an absence of electrical neutrality in the inserted n-layer.

(5) In a semi-infinite structure. 
doped Si n-layer: $\quad d_{\mathrm{n}}=0.40(0.43) \mu \mathrm{m} ; \quad 0.80(0.86) \mu \mathrm{m} ; \quad 1.60(1.70) \mu \mathrm{m} ; \quad$ and $2.60(2.64) \mu \mathrm{m}$. The actual fabrication values are in the parentheses. One of the two highly doped external $\mathrm{n}^{+}$-layers is the original $200 \mu \mathrm{m}$ substrate and the other is the upper thin layer of $0.2 \mu \mathrm{m}$ (see Fig. 4). Taking into account the actual doping level, one can verify that this thickness does not disturb the equilibrium concentration in the volume of the upper thin $\mathbf{n}^{+}$layer. Thus, what differentiates our four groups of samples from the electrical viewpoint is only the potential barrier shape and height and the total accumulated charge (under the same applied voltage) within the inserted layer.

3.4 GEOMETRICAL FACTORS. - In the case of a simple homointerface the geometrical factor can be represented by the extension of the structural transition zone which directly influences the composition of the free carrier space charge [14]. The anisotropic fractions disappear with a transition zone increase. This aspect is not very important in the case of short-range unipolar interactions but becomes significant in long-range bipolar interactions [32]. The size of the effect can be estimated within a modification of the carrier fraction distributions (as a function of the normalized electric field $F_{s}$; see Fig. 5a).
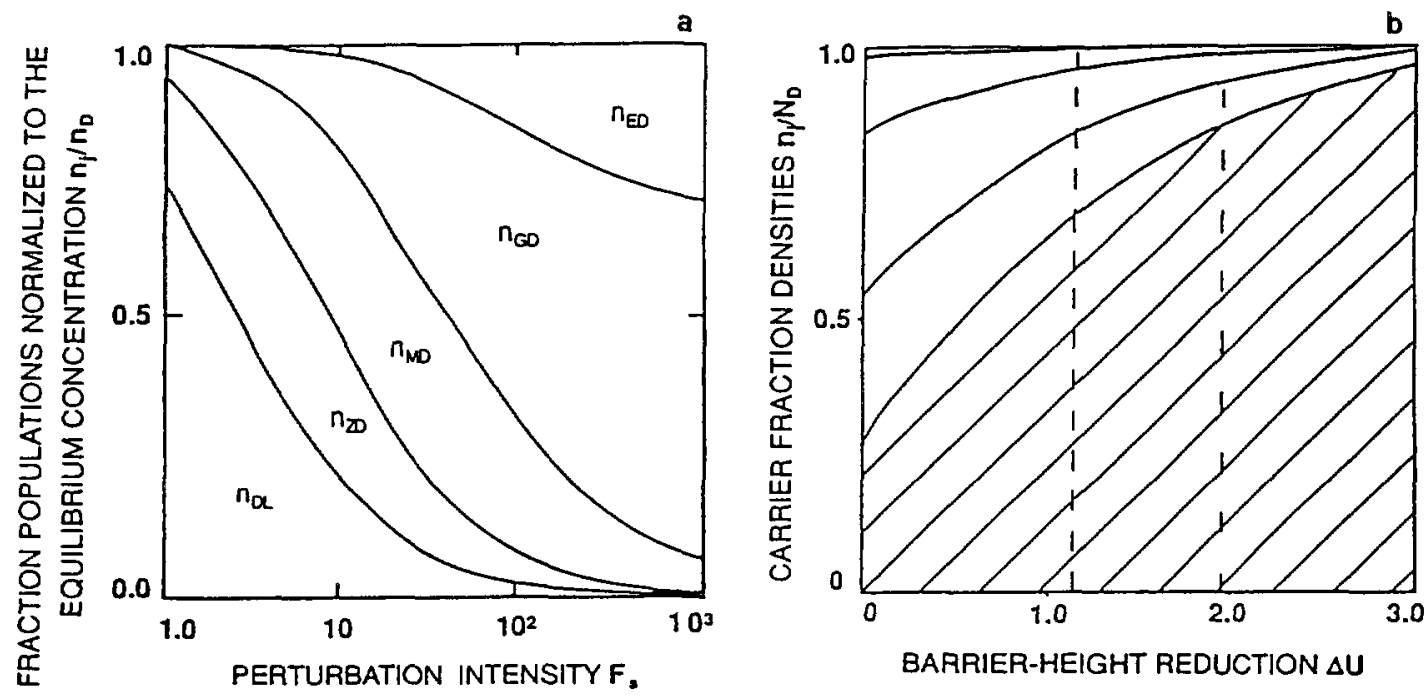

Fig. 5. - Modification of the carrier fraction distributions as a function of the a) normalized electric field $F$, and b) potential barrier reduction $\Delta U$ for a sample with doping ratio $N=81.82$.

The second feature of the geometrical factor concerns the spacing of the two (simple) homointerfaces. On the basis of the analytical representation of the $I(V)$ relation developed for the thermionic emission current only, one can define three factors possessing a geometrical reference. The corresponding formulae can be simplified to a form depending only on the potential barrier reduction $\Delta U$ and the three geometrical factors $\alpha, \beta_{\mathrm{L}}$ and $\beta_{\mathrm{H}}$ as follows

total current

$$
l=S \alpha \mathrm{e}^{\Delta U}\left(\mathrm{e}^{\Delta U}-1\right)
$$

total voltage

$$
V=\left(\frac{k T}{q} \frac{1}{\beta_{\mathrm{L}}}+\frac{\mathrm{e}^{د U}}{\beta_{\mathrm{H}}}\right)\left(\mathrm{e}^{د U}-1\right)+\frac{k T}{q} \Delta U
$$


where $S$ is the junction area. Also the

current geometrical factor

$$
\begin{aligned}
& \alpha=\frac{q^{2}}{\varepsilon \varepsilon_{0}} \mu\left(n_{\mathrm{DL}}\right)^{2} d_{n} \\
& \beta_{\mathrm{L}}=\frac{\varepsilon \varepsilon_{0}}{q} \frac{k T}{q} \frac{1}{n_{\mathrm{DL}}} \frac{1}{d_{\mathrm{n}}^{2}} \\
& \beta_{\mathrm{H}}=\frac{\varepsilon \varepsilon_{0}}{q} \frac{N}{n_{\mathrm{DL}}} \frac{1}{d_{\mathrm{n}} d_{\mathrm{n}^{+}}}
\end{aligned}
$$

where $\mu$ is the majority carrier mobility, $n_{\mathrm{DL}}$ is the equilibrium concentration in the low region and $d_{\mathrm{n}}$ and $d_{\mathrm{n}^{+}}$are the low region and high region widths, respectively. In a thick sample the low region factor

$$
\beta_{\mathrm{L}}=\left.\left(\frac{L_{\mathrm{DL}}}{d_{\mathrm{n}}}\right)^{2}\right|_{d_{\mathrm{n}} \rightarrow \infty} \rightarrow 0
$$

which means that it describes a relation between the extrinsic Debye length $L_{\mathrm{DL}}$ and the inserted layer width $d_{\mathrm{n}}$. For very thick layers this factor loses its physical sense considering the thermionic emission current. Samples having a thickness of the order of the extrinsic Debye length or less are characterized by a stronger thickness dependence on the spillover phenomenon $[7,8]$. If the highly doped regions are sufficiently thick, to keep the equilibrium part, the spillover can be taken into account by replacing $n_{\mathrm{DL}}$ and $N$ in equations (6) and (7) by the experimental spillover values : $n_{\mathrm{DL}}^{*}, N^{*}$ as follows

$$
\beta_{\mathrm{L}}=\frac{\varepsilon \varepsilon_{0}}{q} \frac{k T}{q} \frac{1}{n_{\mathrm{DL}}^{*}} \frac{1}{d_{\mathrm{n}}^{2}} \quad \text { and } \quad \beta_{\mathrm{H}}=\frac{\varepsilon \varepsilon_{0}}{q} \frac{N^{*}}{n_{\mathrm{DL}}^{*}} \frac{1}{d_{\mathrm{n}} d_{\mathrm{n}^{+}}}
$$

where $n_{\mathrm{DL}}^{*}$ is the quasi-equilibrium excess carrier concentration and $N^{*}=n_{\mathrm{DH}} / n_{\mathrm{DL}}^{*}$ is the free carrier concentration ratio [8]. Corresponding theoretical distributions for abrupt Si homointerfaces are shown in figure 6.

Geometrical factors of our four samples have been adjusted on the basis of a numerical procedure that takes into account simultaneously the $I(V)$ characteristics, threshold voltages, actual geometrical dimensions, total charges of confined accumulation layers and the theoretical thermionic current relation. The starting values for the inserted n-layer thickness have been estimated on $\mathrm{C}-\mathrm{V}$ majority carrier profiles. The values thus obtained were then compared with those derived from detailed computations of the actual volume occupied by the space charge of the confined accumulation layer. This procedure, which takes into account several types of sample, was found to give results in excellent agreement with different electrical measurements. The study has confirmed also the inevitable occurrence of a stationary spillover phenomenon in the two thinner samples. In the four groups of Si samples, the theoretical computations of the thermionic emission were performed with the same values for certain parameters, namely $d_{\mathrm{n}^{+}}=200 \mu \mathrm{m} ; \quad L_{\mathrm{DL}}=1.2520 \times 10^{-5} \mathrm{~cm}$; $\varepsilon \varepsilon_{0}=1.0625 \times 10^{-12} \mathrm{Fcm}^{-1} ; \mu_{\mathrm{n}} \approx 1000 \mathrm{~cm}^{2} / \mathrm{Vs}$. Parameters differing from one sample to another are listed in table I.

We notice first that for a critical thickness of the inserted layer $d_{\mathrm{n}}<2 L_{\mathrm{DL}}$, even in the absence of an applied field, a stationary spillover occurs in the majority carriers [7] (upper curves in Fig. 4a). Thus, the validity of the thermionic emission model must be assessed by the existence of the diffusion-drift phenomenon, which is an important component of the majority current under small polarizations. 


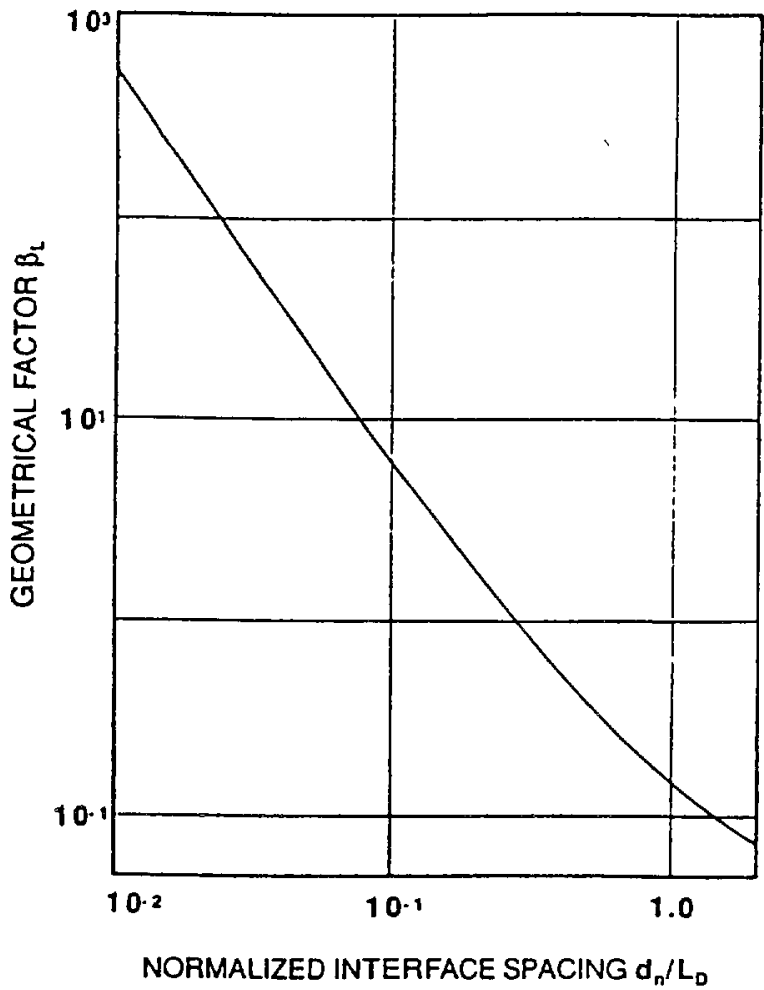

Fig. 6. - Distribution of the geometrical factor $\beta_{\mathrm{L}}$ in the case with one only free carrier fraction as a function of the normalized interface spacing $d_{\mathrm{n}}$ for abrupt $\mathrm{Si}$ homointerfaces where $n_{\mathrm{DL}}=1.1 \times 10^{15} \mathrm{~cm}^{-3}$ and $n_{\mathrm{DH}}=5 \times 10^{15} \mathrm{~cm}^{-3}$

Table I. - Geometric factors and parameters varying from one sample to another in the computation of the thermionic emission current.

\begin{tabular}{|c|c|c|c|c|c|}
\hline \multirow{2}{*}{$\begin{array}{c}\text { State at } \\
\text { the middle } \\
\text { of the } \\
\text { n-layer }\end{array}$} & \multirow{2}{*}{$\begin{array}{c}\text { n-layer } \\
\text { thickness } \\
d_{\mathrm{n}} \\
{[\mu \mathrm{m}]}\end{array}$} & \multirow{2}{*}{$\begin{array}{c}\text { Min. free car. } \\
\text { concentration } \\
n_{\mathrm{DL}}^{*} \\
{\left[10^{15} \mathrm{~cm}^{-3}\right]}\end{array}$} & \multicolumn{3}{|c|}{ Geometrical factors } \\
\hline & & & $\begin{array}{c}\alpha \\
{\left[10^{4} \mathrm{Acm}^{-2}\right]}\end{array}$ & $\beta_{\mathrm{L}}$ & ${ }^{2}{ }^{\beta_{H}}$ \\
\hline Electric neutrality & 2.64 & $1.10=N_{\mathrm{DL}}$ & 0.7716 & 0.2249 & 0.2429 \\
\hline Low spillover & 0.86 & 2.42 & 1.2166 & 0.9634 & 0.1541 \\
\hline High spillover & 0.43 & 8.60 & 7.6820 & 1.0844 & 0.0244 \\
\hline
\end{tabular}

There is one unexpected result of the spillover phenomenon (imposed by the geometrical factor), i.e. an evolution of the fraction distribution inside the accumulated charge. The anisotropic fractions are not modified up to spacing of $2 \times L_{\mathrm{DL}}$ (two extrinsic Debye lengths) when $\beta_{\mathrm{L}} \leqslant 1 / 4$. Shorter spacing brings about an increase of isotropic oscillations. We conclude that two space charges of free carriers formed only by an adjustment of two parameters, i.e. interface spacing and doping level, can represent different fine electric structures. 


\section{Two-homointerface structure in the steady-state.}

4.1 POTENTIAL DISTRIBUTION. - The basic phenomenon of multilayer unipolar conductivity depends on an alternating sequence of highly and lightly doped regions. In our study of four groups of samples with the three-layer $n^{+}-n-n^{+}$structure $\left({ }^{6}\right)$, the conduction current is determined by accumulation properties [8, 49]. The influence of the geometrical factor on the potential barrier shape governing small level injections in particular has been theoretically analyzed in [5] on the ground of the stationary state. With only a decrease of n-layer thickness, the barrier height diminishes and changes its form as a consequence of stationary spillover (see Fig. 3). At higher injection levels, barrier reduction prevails [8]. Concluding, one can observe that the multilayer electric properties are much more nonlinear than would be expected from only the analysis of thermionic emission phenomenon.

The barrier majority conduction is affected by phenomena related to the two geometrical factors namely the effective barrier height which is a result of the spillover phenomenon (stationary-state) and the effective barrier thickness imposed by the inserted layer width which can be sufficiently small to provoke the tunnel effect (steady-state). In this situation the inserted layer properties (actual doping level, majority carrier type [6, 7]) become of secondary importance. The barrier minority conduction (through a one layer or multilayer structure) is largely dependent on the subtle structure of the barrier space charge and its modification under bias.

4.2 Dc-VOlTAGE BIAS. - Many properties of the macroscopic unipolar transport can be deduced from conventional measurements of the conduction current. But this kind of steadystate characterization in a forward regime can be deformed by sample heating. After a stationary-state characterization, all samples were prepared (cutting, set-up. contacts) and encapsulated in typical Schottky diode boxes. Normally, the experimental values of the conduction current exceed the thermionic emission current (computed according to [8]). For the case of a dc-bias mode, conductance is governed in the first place by the diffusion-drift and afterwards also by the heating effect. So the measurements were carried out to reveal separately both diffusion-drift and thermionic emission phenomena as a function of the geometrical factor. In the case of very low forward biases (negative relative to the upper thin layer), the parabolic type nonlinear characteristic already studied by Prochorov et al. [18] occurs and is shown in the insert of figure 7.

The nonlinear diffusion-drift phenomenon is preeminent and causes a current parallel to the thermionic emission current taken into account by Sabnis [50] in his equivalent circuit of the low biased abrupt $n^{+}-n$ junction. By adjustment of the geometrical configuration (obtained from the geometrical factors) of the two upper constituent layers simultaneously, the two phenomena, i.e. accumulation and depletion can be observed together.

Two $I(V)$ characteristics of a shallow junction can be compared by biasing the same $\mathrm{n}^{+}-\mathrm{n}$ $\mathrm{n}^{+}$sample first negatively (Fig. 7b) and then positively (Fig. 8b). In this way it is possible to obscure the diffusion-drift current and to observe only the thermionic emission phenomenon of the deep junction. Figure 7 illustrates the diffusion-drift phenomenon in the case of any modification of the corresponding quasi-neutral concentration in the thin $\mathrm{n}^{+}$-layer whose thickness has been intentionally kept small (recall that $d_{\mathrm{n}^{+}}=0.2 \mu \mathrm{m}$ ). The thickness ratio of the upper thin $\mathrm{n}^{+}$-layer relative to the extrinsic Debye length of this region equals 14.742 $\left(0.2 \mu \mathrm{m} / 1.3567 \times 10^{-2} \mu \mathrm{m}\right)$. In the stationary-state there always exists a bulk part of the thin

$\left(^{6}\right)$ This is in contrast to the case of the inverse three-layer profile as in the $n-n^{+}-n$ structure of GaAs considered in [26], where the depletion properties are predominant. 


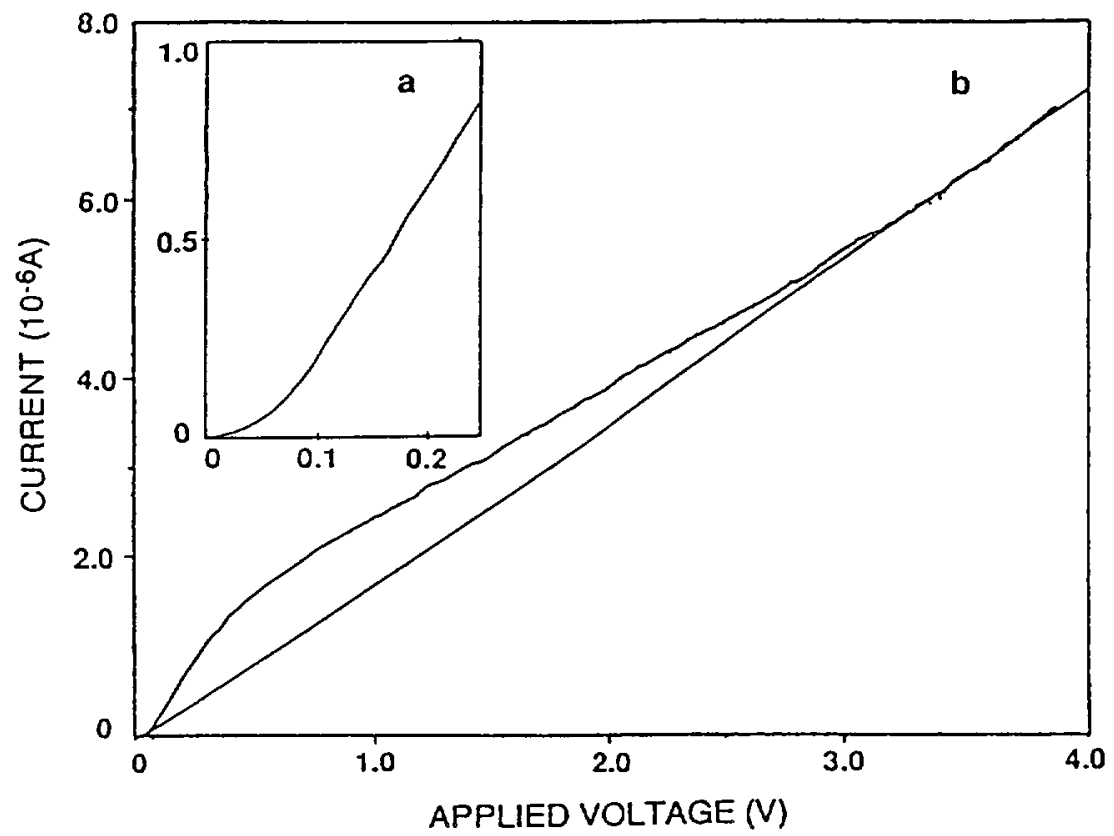

Fig. 7. - Diffusion-drift effect on the experimental characteristic $I(V)$ of the symmetrical, $\mathrm{n}^{+}-\mathrm{n}-\mathrm{n}^{+}$two homointerface structure in : a) a very low forward bias regıme where $I(V)$ has a parabolic shape [18] ; b) an expanded forward bias regime. The nonlinear phenomenon of diffusion-drift is predominant (experimental curve) resulting in the occurrence of a current parallel to the almost linear thermionic emission current (theoretical curve).
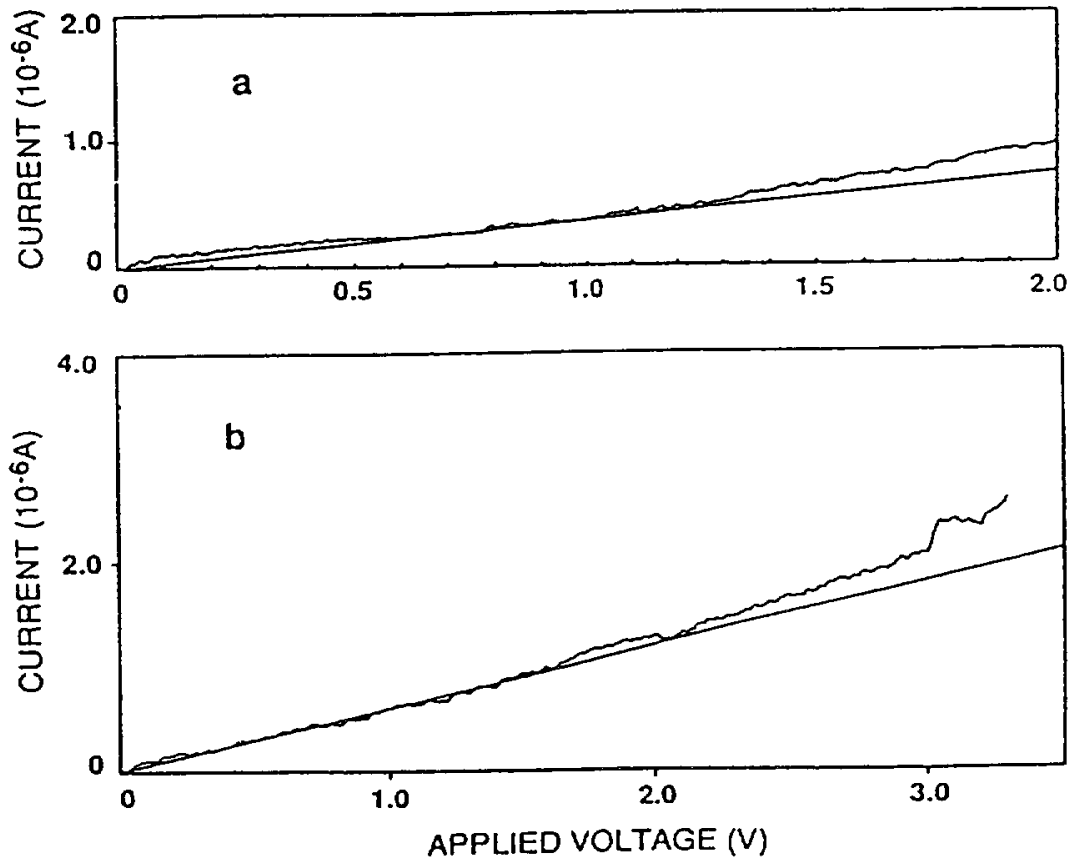

Fig. 8. - Experimental characteristic $I\left(V^{r}\right)$ obtained in the steady-state for symmetrical two homointerface structures $n^{+}-n-n^{+}$exhibiting only the thermionic emission phenomenon, the diffusion-drift being suppressed by the evolution of the total resistance of the positıvely biased superficial layer. 
$\mathbf{n}^{+}$-layer which maintains its electrical neutrality. In figure $8 \mathrm{~b}$ the diffusion-drift is completely absent in the $I(V)$ characteristic (which becomes practically linear) of the three layer structure only by means of a bias which allows proportional emptying of the upper thin $\mathrm{n}^{+}$-layer of majority carriers. In other words, the electrically neutral volume of this layer vanishes under the effect of applied reverse bias. In practice, the diffusion drift phenomenon, being a function of the geometrical factor, always exists but its importance depends on the interface spacing. Figure 9 shows a comparison of the bias thresholds for samples with and without short-range interactions.

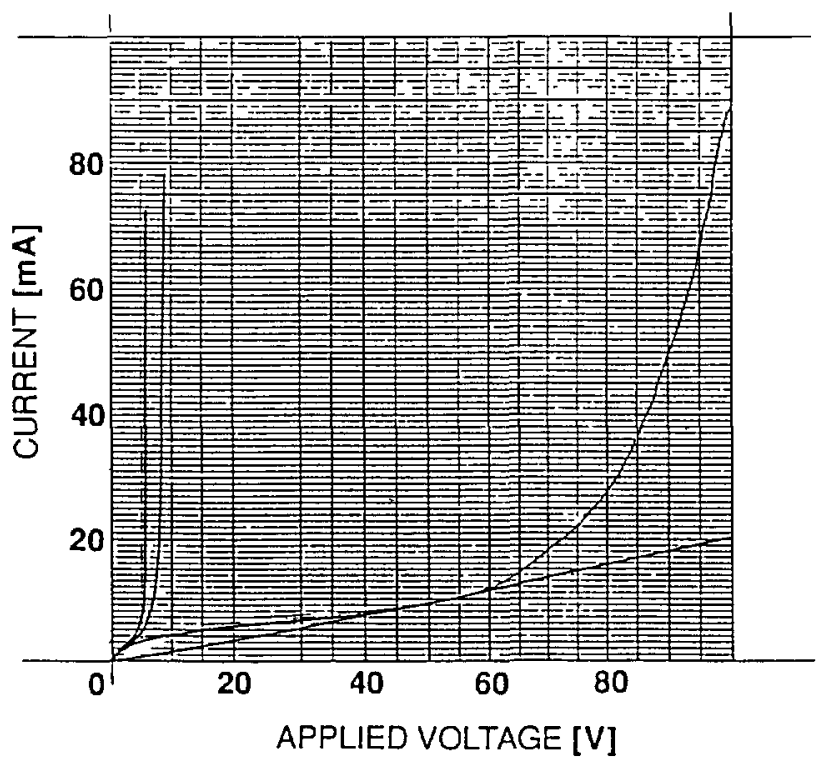

Fig. 9. - Disposition of strong field nonlinearity for three different interface spacings showing the importance of the geometrical factor. The comparison of the bias thresholds for samples with and without short-range interactions allows perceiving of the beginning of the tunnel effect.

At the highest bias, the conventional dc-current measurements may cause a thermal breakdown. To avoid heating in the neighborhood of the interface, our steady-state measurements (in the usual dc-regime) were limited to only very low bias, $E_{\text {eff }}<15 \mathrm{~V} / \mathrm{cm}$, and low bias, $15 \mathrm{~V} / \mathrm{cm} \leqslant E_{\text {eft }} \leqslant 200 \mathrm{~V} / \mathrm{cm}$, and a small contact area. We have used electrodes whose active contact areas were about $5 \times 10^{-9} \mathrm{~cm}^{-2}$

If the applied polarization is relatively important, the usual steady-state $I(V)$ characteristic will reveal the thermionic emission phenomenon depending only on the potential barrier height. In this case the carrier concentration reference level at the n-layer is bias dependent. Here, we would like emphasize that a simple analytical relation between the conduction current and applied voltage together with the three geometrical factors and the barrier reduction [Eqs. (3) and (4)] published in our previous paper [8] gives practically the same $I(V)$ distribution as the more sophisticated theory presented in [9].

4.3 PUlSED VOLTAGE BIAS. - Two groups of measurements of the $I(V)$ characteristic were carried out at room temperature : conventional steady-state measurements where considerable heating has to be taken into account (even at very low bias) and pulsed regime measurements where heating can be totally neglected. All bias regimes (from hundredths to hundreds of volts) 
can be envisaged only in quasi-steady-state measurements. The experimental variations related to the three principal geometrical factors (taking into account interface spacing and the doping levels of all regions) can be deduced from the three curves in figure 9 which represent the conduction current as a function of the applied voltage for three different interface spacings. By comparison, figures 9 and 10 show the theoretical curves (practically straight lines) of our numerical model for the thermionic emission effect. The study of the $I(V)$ characteristics of our samples allows us to distinguish five bias intervals [8] where only two (first and fourth) correspond to the thermionic emission current. A comparison of the stationary spillover (without bias) with the steady-state spillover (with bias) is depicted in figure 11 by the two upper curves. The same phenomenon has already been reported by the van der Ziel group [7]. Figures $10 \mathrm{a}$ and $\mathrm{b}$ shows the result obtained with the smallest spacing $\left(d_{\mathrm{n}}=0.43 \mu \mathrm{m}\right)$ where the thermionic emission range decreases drastically.
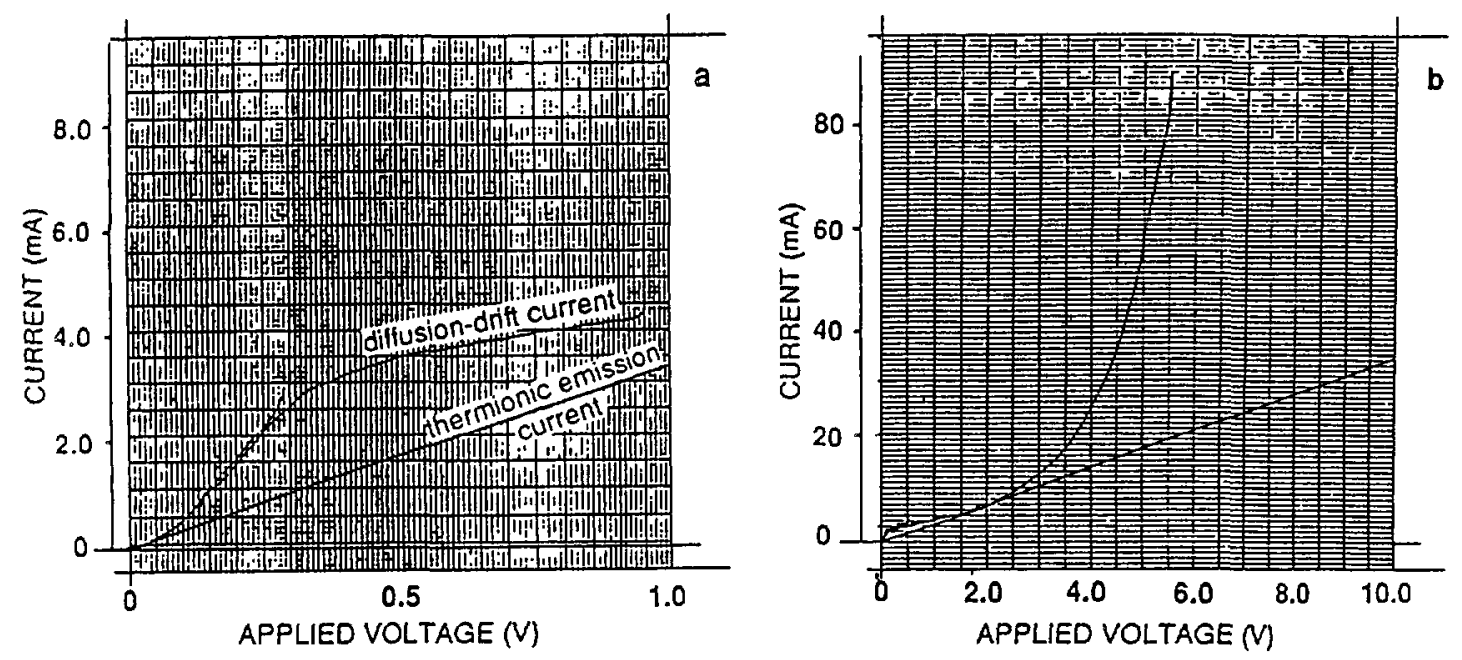

Fig. 10. - Experimental characteristic $I(V)$ of a symmetrical two homointerface structure $\mathrm{n}^{+}-\mathrm{n}-\mathrm{n}^{+}$ obtained in the quasi-steady-state pulsed regime. Thickness of the inserted layer $d_{\mathrm{n}}=0.43 \mu \mathrm{m}:$ a) low bias ; b) maximal real bias.

In very high biases (fifth interval : $E_{\text {appl }} \geqslant 3.0 \times 10^{4} \mathrm{~V} / \mathrm{cm}$; Tab. II) the current largely surpasses the component due to the thermionic emission. This behavior can be accounted for by the tunnel effect. The importance of the geometrical factor on the tunnel effect may be estimated by the appearance of a strong field nonlinearity for three of the interface spacings (see Fig. 9). The range of the thermionic emission bias decreases greatly in the case of the thinnest sample being covered by the tunneling (Fig. 10). At the threshold voltage the n-layer is only partially filled with excess carriers.

The magnitude of the activating bias (strongly dependent on the geometrical factor, i.e. inserted layer thickness) can be estimated on the basis of $I(V)$ characteristics in logarithmic coordinates (see Tab. II and Fig. 9). For the thickest sample $\left(d_{n}=2.64 \mu \mathrm{m}\right)$ this voltage can be estimated at $32.0 \mathrm{~V}$. This means that the effective electric field is $E_{\text {appl }} \approx 3.5 \times$ $10^{4} \mathrm{~V} / \mathrm{cm}$. In the case of the thinnest sample $\left(d_{\mathrm{n}}=0.43 \mu \mathrm{m}\right)$ these values are respectively $1.3 \mathrm{~V}$ and $3.0 \times 10^{4} \mathrm{~V} / \mathrm{cm}$. In the center of the n-layer the effective electric fields are stronger because the high resistivity part is reduced in width by the accumulated layer $[6,9]$. So the 


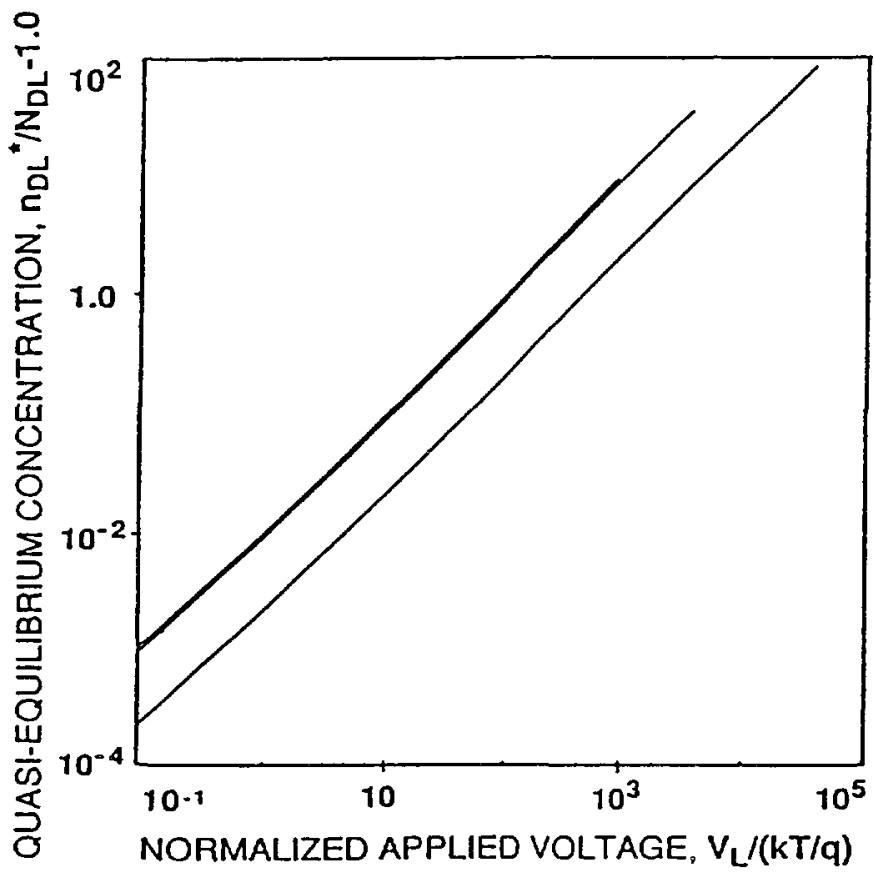

Fig. 11. - Stationary spillover (without bias) and steady-state spillover (with bias) depicted by the two upper curves. Comparison (lowest curve) is made using the results derived from a structure that consists of an electrically neutral zone (thick layer).

Table II. - Tunneling thresholds.

\begin{tabular}{|c|c|}
\hline$d[\mu \mathrm{m}]$ & $E_{\mathrm{appl}}\left[10^{4} \mathrm{~V} / \mathrm{cm}\right]$ \\
\hline 0.43 & 3.02 \\
2.64 & 3.50 \\
\hline
\end{tabular}

Note: In our experimental approach we can neglect to a first approximation the modification of the strong field mobility because its change is opposite to the evolution of the observed conduction.

intensity of the effective field can be estimated as $3.0 \times 10^{4} \leqslant E \leqslant 2.5 \times 10^{5} \mathrm{~V} / \mathrm{cm}$. We have to mention two other transport mechanisms that depend strongly on the geometrical factor, i.e. tunneling and ballistic transit which are responsible for very large current densities for small interfacial spacing. It follows that in figure 9 the rapid current increase is due to the tunnel effect of the non ballistic transport. Among the causes likely to induce a modification of the voltage thresholds bearing on this effect, the possibility of the space charge being stored by the applied voltage in the most resistive inserted layer has been retained. The analysis of this phenomenon is another subject and will not be treated in this paper. 


\section{Conclusions.}

Concluding, one can observe that the multilayer electric properties are much more nonlinear than would be expected from only the analysis of thermionic emission phenomenon. The macroscopic transport measurements carried out on thin three layer $n^{+}-n-n^{+}$homostructures at ambient temperature reveals two considerably different behaviors depending on the interface spacing. This enables us to distinguish phenomena that account for the free carrier space charge. The potential barrier governs the steady-state characteristics at small and moderate injection levels. With a decrease of the interface spacing, the barrier height diminishes and changes form as a consequence of the stationary spillover.

The same total space charge can be composed of different free carrier fractions that depend on the interface abruptness, interface spacing, electric neutrality disturbance and doping ratios. So the geometrical factor combining with the electrostatic forces may influence the nature of the thermal micromovement (microscopic oscillations) of the confined majority carriers. The interface spacing modifies the fraction composition of the accumulated majority carriers : the anisotropic fractions are not concerned up to a distance equal to two extrinsic Debye lengths, $\left(2 \times L_{\mathrm{DL}}, \beta_{\mathrm{L}} \geqslant 1 / 4\right)$. Shorter interface spacing provokes modification of anisotropic oscillations and the anisotropic fractions disappear rapidly (see Fig. 5). So there is a kind of equivalence between steady state polarization and geometrical factors (interface abruptness and spacing) and, as a consequence, the minority carrier recombination properties.

The nature of the majority carrier oscillations affects the minority carrier micromovement. The results can be observed on the long range interface interaction that translates the evolution of the minority carrier recombination mechanisms and their lifetime. This aspect should be studied in details hereafter.

\section{References}

[1] Yu S. Y., Thomas G., Solid-State Electron. 20 (1977) 19-25.

[2] Poorter T., Solid-State Electron. 22 (1979) 311-325.

[3] Zimmermann W., Phys. Status Solidi (a) 12 (1972) 671-678.

[4] Rohatgi A., Rai-Choudhury P., IEEE Trans. Electron. Dev. ED-31 (1984) 596-601.

[5] Kuznicki Z. T.. Electrıcal Properties of Polycrystalline Thin Films (PWN Warszawa-Lodz, 1984) in Polish and Solar Cells 13 (1984) 1-18.

[6] Kim C. K.. Yang E. S., Solld-State Electron. 13 (1970) 1577-1589.

[7] Van der Zıel A., Shur M. S., Lee K., Tzu-Hung Chen, Amberiadis K., IEEE Trans. Electron. Dev. ED-30 (1983) 128-137.

[8] Kuznicki Z. T., Martinez A., Siffert P.. IEEE Trans. Electron. Der. ED-39 (1992) 409-421.

[9] Grinberg A. A., Luryi S., J. Appl. Phys. 61 (1987) 1181-1189.

[10] Lieb D. P., Jackson B. D., Root C. D., IRE Trans. Electron. Dev. ED-9 (1962) 143-153.

[11] Shockley W.. Prim R. C., Phys. Rev. 90 (1953) 753-758.

[12] Kuznicki Z. T., Electrical Properties of Polycrystalline Thin Films (PWN Warszawa-Lods, 1984) in Polish and Thin Solld Films 127 (1985) 269-282.

[13] Fossum J. G., IEEE Trans. Electron. Dev. ED-24 (1977) 322-325.

[14] Kuznicki Z. T., I Appl. Phys. 69 (1991) 6526-6541.

[15] Kuznicki Z. T., in L. Zdanowicz Ed., Proc. 1st Natl. Autumn School on Physics of Thin Films ; Szczyrk. Poland, October 19-25, 1979 (Polısh Scientific Publishers, Wroclaw, 1981) and Thin Solid Films 85 (1981) 169-179.

[16] Davies L. W., Proc. Phys. Soc London B 70 (1957) 885-889.

[17] Gunn J. B., J. Electronics and Control 4 (1958) 17-50.

[18] Prochorov E. D., Szechovcov N. A., Prochorov A. D., Radtotechnika \& Elektronika 9 (1964) 20142021. 
[19] Kuznickı Z. T., Solid-State Electron. 34 (1991) 323-325.

[20] Kuznicki Z. T., Bull. Ac Pol., Tech. 29 (1981) 111-118.

[21] Kennedy D. P., O’Brien R. R., IBM J. Res. Develop. 13 (1969) 212-214.

[22] Klopfenstein R. W., Wu C. P., IEEE Trans. Electron. Dev. ED-22 (1975) 329-334.

[23] Kuznicki Z. T., Zimmermann B., Rev. Phys. Appl. 24 (1989) 851-860.

[24] Ehlers D. H., Mills D. L., Phys. Rev. B 34 (1986) 3939-3947.

[25] Kuznicki Z. T., Rev. Phys. Appl. 23 (1988) 1313-1316.

[26] Shenai K., Dutton R. W., IEEE Trans. Electron. Dev. ED-35 (1988) 590-603.

[27] Shur M. S., Eastman L. F., IEEE Trans. Electron. Dev. ED-26 (1979) 1677-1683.

[28] Rosenberg J. J., Yoffa E. J.. Nathan M. I., IEEE Trans. Electron. Dev ED-28 (1981) 941-944.

[29] Shur M. S., IEEE Trans. Electron. Dev. ED-28 (1981) 1120-1130.

[30] Shur M. S., Proceedings of IEDM, Washington (December 1980) 618-621.

[31] Rohatgi A., Rai-Choudhury P., Meier D. L.. Milstein J. B., Conf. Record 17th IEEE Photovoltaic Specialists' Conf., 1984.

[32] Kuznicki Z. T., Solar Energy Materials and Solar Cells, in press.

[33] Korec J., J. Crystal Growth 46 (1979) 362-370.

[34] Aleksandrov L. N., Thin Solid Films 50 (1978) 13-24.

[35] Nossarzewska-Orlowska E., Lachowski A., Borkowicz J., Electron. Technol. 11 (1978) 47-60.

[36] Korec J., Borkowicz J., Electron. Technol. 10 (1977) 3-15.

[37] Nishida M., IEEE Trans Electron. Dev. ED-26 (1979) 1081-1085.

[38] O'Brien R. R., IEEE Trans. Electron. Dev. ED-27 (1980) 1848-1849.

[39] Baccarani G., Rudan M., Spadine H., Maes H., Vanderworst W., Van Overstraeten R., Soldd-State Electron. 23 (1980) 65-71.

[40] Wilson C. L., IEEE Trans. Electron. Dev. ED-27 (1980) 2262-2267.

[41] Kennedy D. P., Murley P. C., Kleinfelder W., IBM J. Res. Develop. 12 (1968) 399-409.

[42] Kroemer H., Chien W. Y., Solid-State Electron. 24 (1981) 655-660.

[43] Johnson W. C., Panousis P. T., IEEE Trans. Electron. Dev. ED-18 (1971) 965-973.

[44] Kuznicki Z. T., Stareev G., Grabinski J., Strazynska H., Determination of Free Carrier Profiles and Transition Regions in Si Multilayer Structures : Rep. Institute of Electron Material Technology (1985) in Polish.

[45] Morris B. L., J. Electrochem. Soc. 121 (1974) 422-427.

[46] Borkowicz J., Korec J., Nossarzewska-Orlowska E., Phys. Status Solidi (a) 48 (1978) 225-228.

[47] Schuman P. A. Jr, Gardner E. E., J. Electrochem. Soc. 116 (1969) 87-92.

[48] Choo S. C., Leong M. S., Kuan K. L., Solid-State Electron. 19 (1976) 561-565.

[49] Kuznicki Z. T., J. Phys. III France 2 (1992) 2349-2371.

[50] Sabnis A. G., Solid-State Electron. 22 (1979) 667-669. 